\title{
Electromagnetic Field Effect on Skeletal Muscles and Skin of Albino Rat Embryos Histological and Histochemical Studies
}

\author{
Nora Ahmed Obaid Alkaabi \\ Zoology Department, Girls College of Science, King Faisal \\ University,Dammam,K.S.A.
}

\begin{abstract}
Aim of the work :The present study aimed to investigate the possible effect of the electromagnetic field (EMF) on the skeletal muscles and skin of fetuses maternally exposed to EMF.

Material and Methods: Pregnant Swiss albino rats were exposed to EMF $(50 \mathrm{~Hz} \& 2 \mathrm{mT})$ 8 hours day after day 10 days before pregnancy and 20 days, day after day through pregnancy.

Results: Exposure of pregnant rats to the electromagnetic field (EMF)of $50 \mathrm{~Hz}$ and 2 millitesla(2mT), showed many dystrophic changes in the skeletal muscles and skin of their fetuses .Highly thickened and disturbed epidermal layer was noted with decreased number of hair follicles. Some hemorrhagic areas were noted in the dermis. Highly disturbed skeletal muscle fibers were noted with many deleterious changes in the nuclei of their cells. Altered protein content, polysaccharides and collagen bundles were observed in the muscle fibers and skin of fetuses taken from exposed pregnant rats to EMF.
\end{abstract}

Conclusion: It is clear that EMF has a deleterious effect on skin and skeletal muscles of fetuses maternally exposed to EMF.

\section{Introduction}

Children in both industrial and developing countries are exposed to a large variety of environmental agents including indoor and outdoor air pollution, water and food contamination, chemicals (eg,pesticides, lead and mercury), and physical agents such as ultraviolet radiation, excessive noise and electromagnetic fields. Exposure to electric and magnetic fields from $0-30 \mathrm{~Hz}$ has been increasing greatly as countries increase their capacity to generate and distribute electricity and take advantage of many new technologies (Kheifets et al;2005).They added that children have a large lifetime of exposure than adults, and from a physiologic point of view ,they have a developing nervous system ,their brain tissue is more conductive than that of adults because it has a high water content and ion concentration .The skin is the heaviest single organ in the body and it represents about $16 \%$ of the total body weight (Hassan \&Sorour, 2006).

Many authors noticed skin sensitivity to EMFs. In 2001 Imaida et al; noticed relationship between mouse skin carcinogenesis and exposure to1.5 $\mathrm{GHz}$ electrom-agnetic near fields. $\mathrm{Xu}$ et al.
(2001) tried to study the effect of EMFs on muscle fibers of mice and they stated that field intensity of $1 \mathrm{mT}$ might be considered as a threshold level for enhancing muscle circulation. In 1996,Detlavs et al; noticed accumulation of collagenous protein in skin of Wistar rats exposed to53.53GHz.Altered protein expression was noticed in human skin by Karinen et al.(2008)after exposure to radiofrequency modulated electromagnetic fields. Physiological functions of human body are regulated by electric currents, therefore, it is not surprising that placing human body within electromagnetic field of sufficient strength may affect physiological and biological processes and human health (Karinen et al;2008). They added that EMF are considered as potential cause of ailments as sleep disorders, headache or allergy-like Symptoms. Altered glycogen content, protein content and collagenous fibers were noted by Eid and Al-Dossary(2007) in liver of pregnant rats and their fetuses which exposed to $\operatorname{EMF}(50 \mathrm{~Hz}$ and $2 \mathrm{mT})$.

Skin and skeletal muscles were chosen for the present study because skin is considered as a mirror for the internal body 
organs and its healthy look gives a good idea about the state of such organs. Skeletal muscle fibers which are considered as voluntary and striated muscle fibers play an important role in the body.

\section{Material and Methods}

\section{Animal model:}

This work was carried out on Swiss albino rats, 20 pregnant females and 10 males kept for fertilization, each weighs about 160 grams. Animals were housed in plastic cages.

They were kept under normal room conditions of temperature, humidity and normal light cycle and freely supplied with food and water.

\section{Experimental design:}

Female rats were categorized into the following groups :

Group ( 1 ): 10 pregnant rats were kept under normal conditions unexposed to the electromagnetic fields.

Group ( 2 ) : 10 pregnant rats were exposed to alternating electromagnetic field $(50 \mathrm{~Hz})$ with flux density $2 \mathrm{mT}, 8$ hours day after day, for 10 days before pregnancy then 20 days , day after day ,during pregnancy period( the total days of exposure were15 days ), then they were sacrificed at day 20 of gestation.

For alternating electromagnetic fields induction, exposure chamber was designed to obtain alternating current electromagnetic fields of $50 \mathrm{~Hz}$ frequency within it where the rats were placed between the poles of the electromagnet in a well ventilated plastic container which maintained a constant environmental temperature.

\section{Histological and histochemical techniques:}

Fresh small pieces of fetal skin and skeletal muscles were fixed in $10 \%$ neutral buffered formalin solution \& Carnoy's Fluid for histological and histochemical studies. Paraffin sections were prepared 2 $\mu m$ thickness and stained with Harris haematoxylin and eosin [Bancroft and Gamble, 2002]. Polysaccharides were detected by PAS ( periodic acid Schiff ) method [Hotchkiss , 1948]. Proteins were detected by mercuric bromophenol blue method [Mazia et al., 1953]. Collagen was detected by Mallory trichrome stain [Pearse, 1977].

\section{Results}

Normal structure of fetal skin is shown in figs.(1\&2). Exposure of pregnant rats to EMF cause many dystrophic changes in the skin of their fetuses. These changes included: highly thickened epidermis, deformed keratin layer, disturbed and irregular Malpighian layer, congested dermal blood vessels, reduced hair follicles with many hemorrhagic areas in the dermal layer(figs.3-5).

Skin of control fetuses showed dense stain ability of proteinic materials in the epidermal layer and hair follicles with less stain ability in the keratin and the dermal layers (fig.6).Skin of fetuses taken from the exposed mothers (exposed group) showed decreased stain ability for total proteins in the keratin and epidermal layers with dense stain ability in the RBCs of hemorrhagic areas (fig.7).

Intense reaction for polysaccharides was observed in the medulla of some hair follicles and the hypodermal muscle fibers of the control fetal skin with less stain ability in the epidermal and dermal layers. Poor stain ability was observed in the keratin layer(fig.8).Densely aggregated polysaccharide granules were observed in the highly thickened fetal skin of the exposed group, while some areas of the epidermis appeared thin and poorly stained and so the keratin layer(figs.9\&10).

Normal distribution of collagen fibers in the skin of control fetus is shown in fig.(11).Keratin accepted deep blue coloration with thin collagen fibers in the basement membrane and throughout the dermal layer. The superficial dermal layer contained fine collagen bundles, beneath it the thicker, coarse deeper reticular dermis contained large compact collagen fibers. Figs.(12-14)showed increased collagen fibers in the: highly thickened keratin layer, epidermal and dermal layers, around the hypodermal muscle fibers . Fetal skeletal 


\section{Nora Ahmed Obaid Alkaabi}

muscle fibers of the control group is shown in fig. (16).Disturbed and discontinuous skeletal muscle fibers could be observed in the exposed group. Some nuclei were not surrounded by the cytoplasm, others accepted dark blue coloration (pyknosis). Fig.(17)showed the normal distribution of total proteins in the fetal skeletal muscle fibers of the control group.Hypostainability could be observed in the fetal skeletal muscle fibers of the exposed group. Most nuclei were faintly stained, others showed hyperstainability (fig.18).Densely stained polysaccharides were noticed in the fetal skeletal muscle fibers of the control group (fig.19). Highly decreased polysaccharides could be observed in the fetal skeletal muscle fibers of the exposed group (fig.20). Normal distribution of collagen fibers in the fetal skeletal muscle fibers of the control group is shown in fig.(21).These fibers were noticed in between normal fetal skeletal muscle fibers. Increased collagen bundles are noticed in between and around the discontinuous muscle fibers of fetuses taken from exposed rats .Increased collagen fibers were also observed in and around the walls of the dilated blood vessels (fig.22).
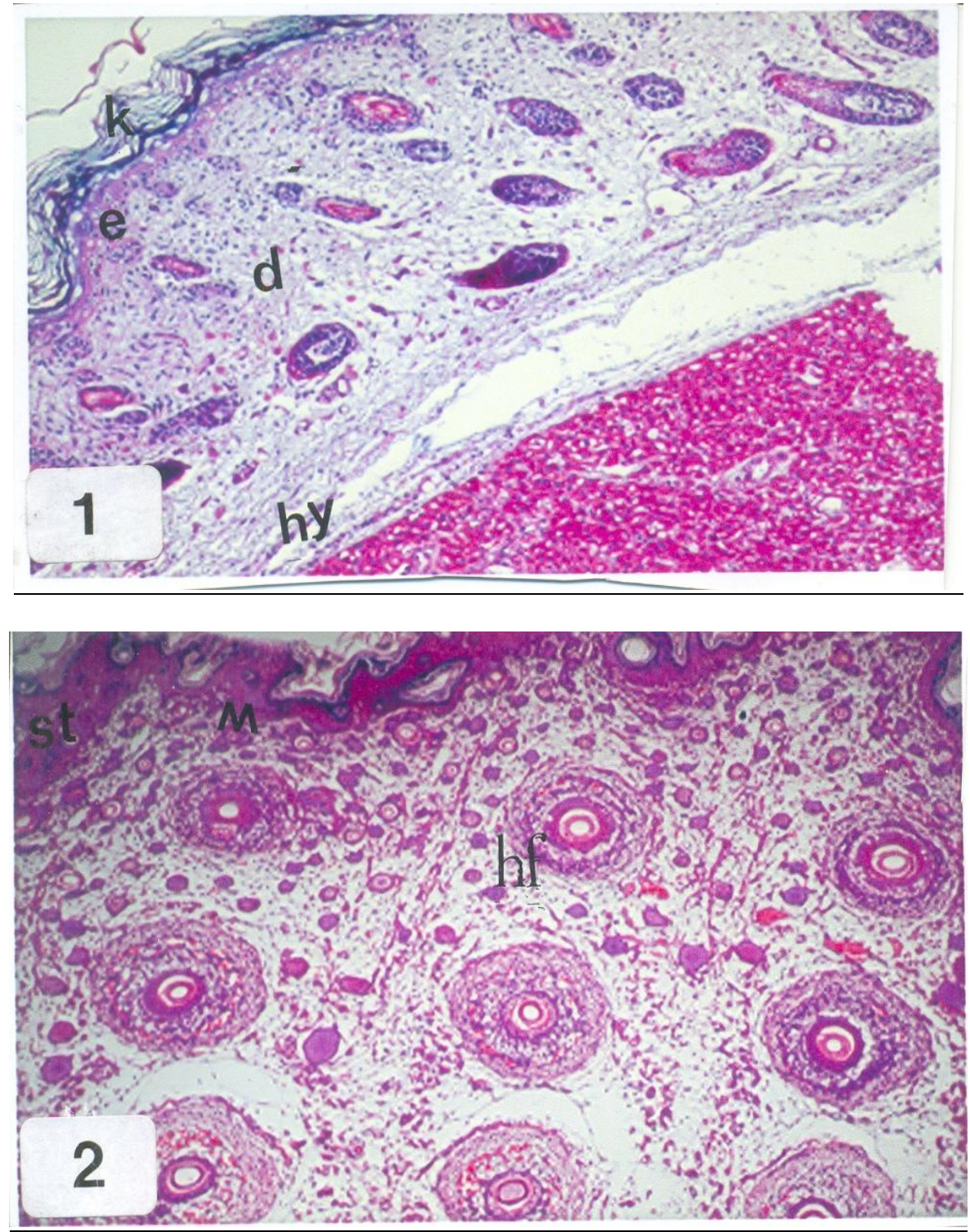

Figs.(1\&2): Photographs of sections in the control fetal skin showing:normal structure of epidermis (e), dermis (d) and hypodermis (hy). Notice keratin (k), stratified squamosal epithelial cells (st), Malpighian layer (M) and well developed hair follicles (hf)

(H\&EX200) 


\section{Electromagnetic Field Effect on Skeletal Muscles and...........}
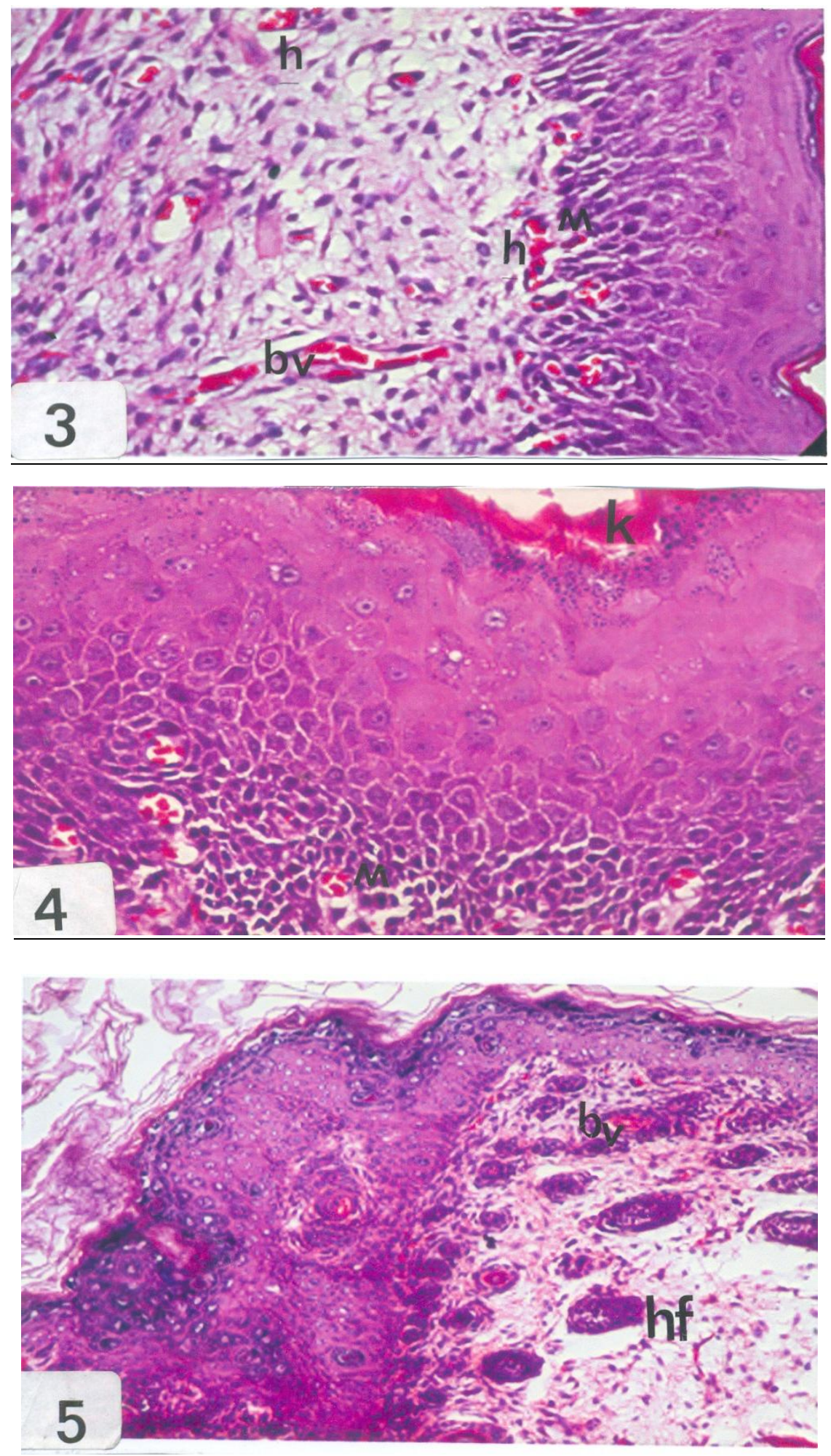

Figs.(3-5) Photographs of sections in the fetal skin maternally exposed to EMF (exposed group).Notice: thickened epidermal layer with deformed keratin layer(k),disturbed and irregular Malpighian layer(M),highly dilated and congested blood vessels(bv),some areas of the dermal layer appeared devoid of hair follicles with many hemorrhagic areas in the dermis(h).

( Figs .3\&4 H\&EX40)

Fig.(5) Notice :highly disturbed and irregular keratin and epidermal layers,atrophied hair follicles (hf),irregular hair follicles inside the epidermal layer, congested and dilated blood vessels(bv),small hemorrhagic areas in the dermis and highly thickened epidermis.

(H\&EX200) 


\section{Nora Ahmed Obaid Alkaabi}
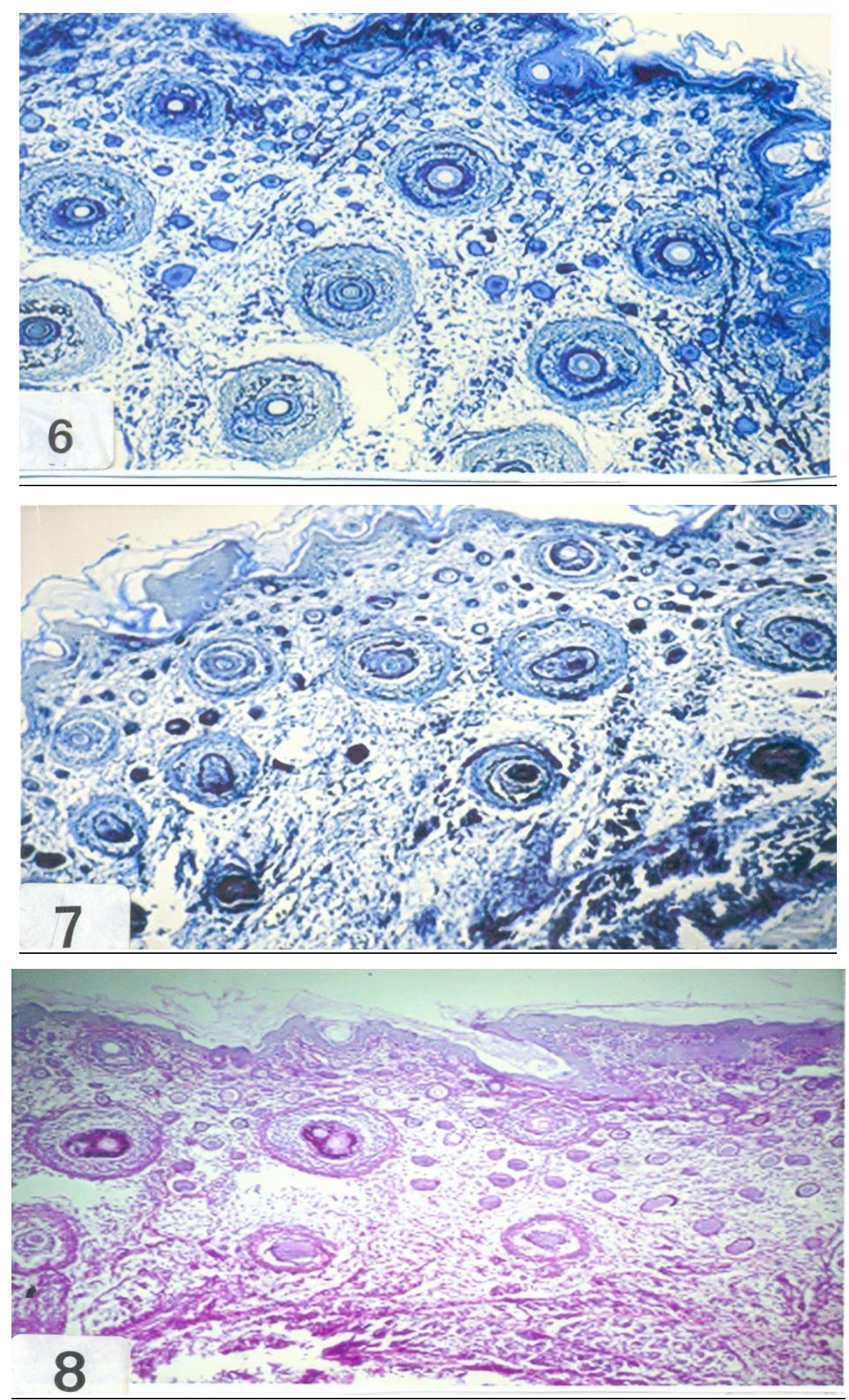

Fig.(6) : Showing control fetal skin with normal distribution of total proteins. Dense stain ability is observed in the epidermal layer and hair follicles but less stain ability is detected in the keratin and dermal layers.

(Mercuric bromophenol blueX200)

Fig (7):Showing decreased stain ability for total proteins in the keratin and the epidermal layers of a fetus taken from exposed rat. Dense stain ability can be observed inRBCs of the hemorrhagic areas

(Mercuric bromophenol blueX200).

Fig.(8): Showing polysaccharides in skin of control fetus .Notice intense reaction in the medulla of some hair follicles and hypodermal muscle fibers with less stain ability in the epidermis and dermis.Poor stain ability can be observed in the keratin layer

(PASX200) 

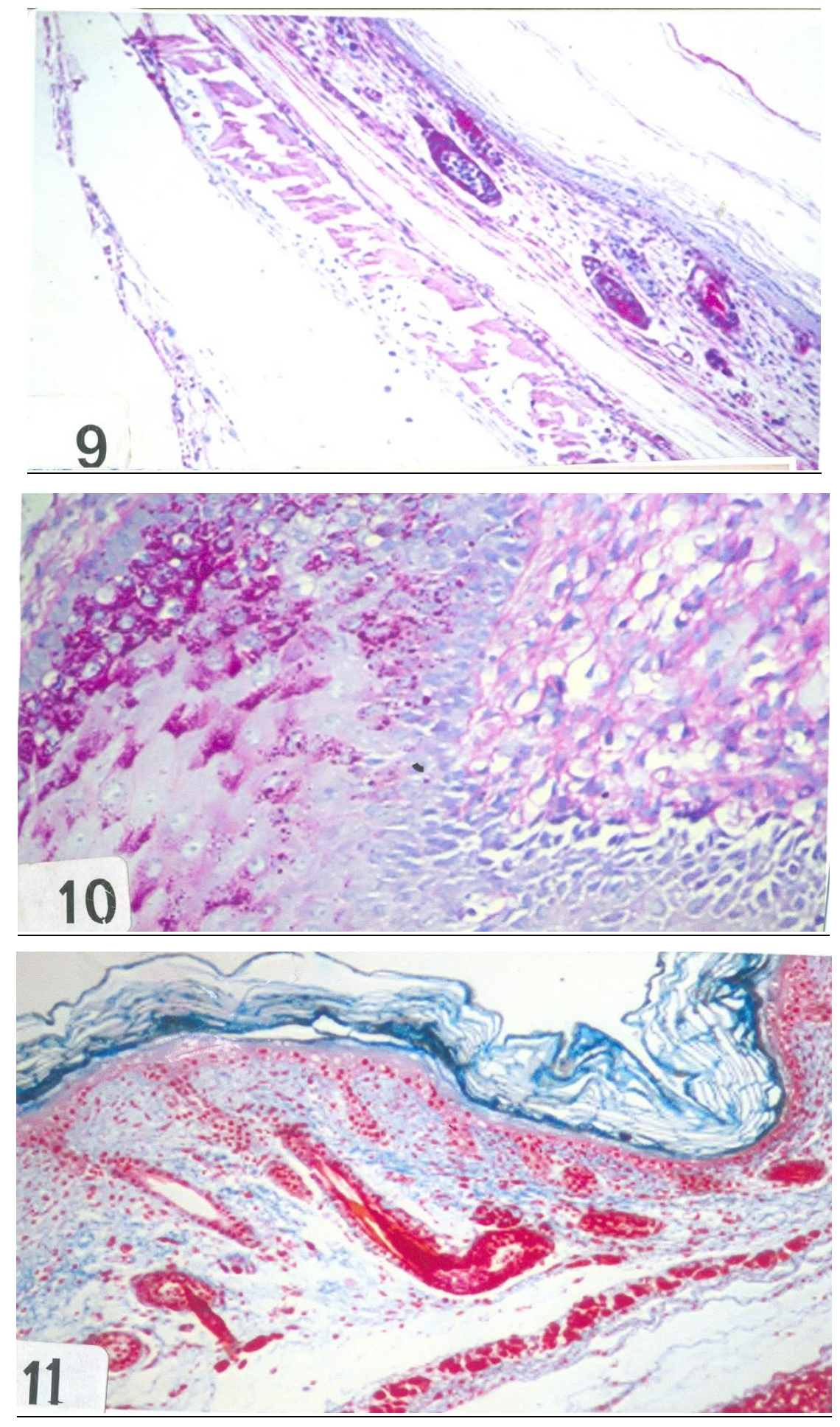

Figs.(9\&10): Showing distribution of polysaccharides in the skin of a fetus taken from exposed rat.

Fig.(9): Notice:poor stain ability in the keratin layer and epidermis .Hair follicles in the dermal layer appeared densely stained, less stain ability could be observed in the hypodermal muscle fibers.

Fig.(10):Showing aggregated polysaccharide granules in the highly thickened epidermal layer.Malpighian layer appeared poorly stained and the dermal layer is moderately stained (Fig.9 PASX200\&Fig.10 PASX400) .

Fig.(11):Showing normal distribution of collagen bundles in the control fetal skin.Notice:deep blue coloration in the keratin layer ,thin bundles in the basement membrane and throughout the dermal layer and around the hypodermal muscle fibers.

(Mallory trichrome stainX200) 

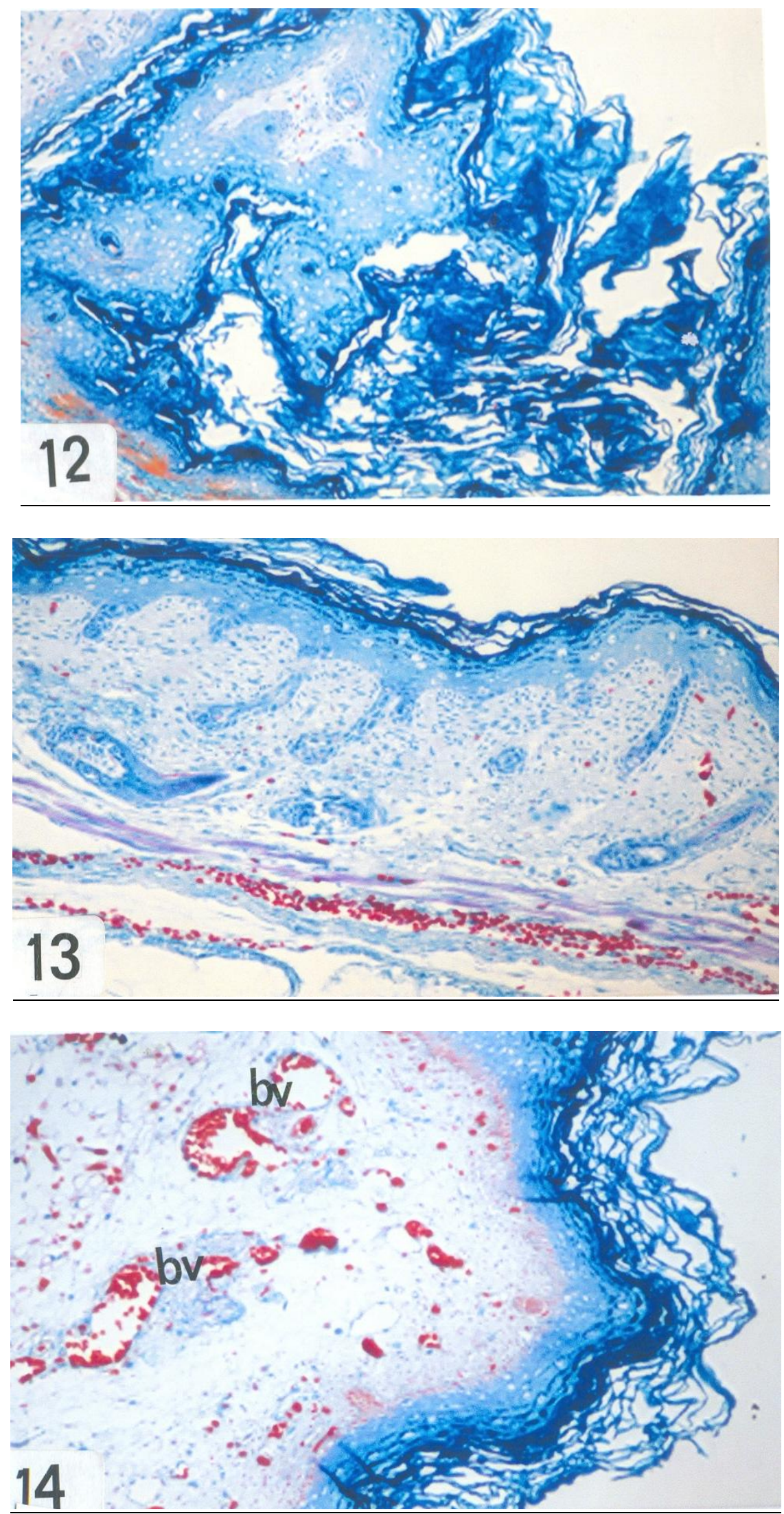

Figs.(12-14):Showing highly thickened and disturbed keratin layer which accepted deep blue coloration with increased collagen bundles in the epidermis ,dermis ,around the hypodermal muscle fibers, in the cortex and medulla of hair follicles and around walls of the dermal blood vessels (bv). (Mallory trichrome stainX200) 

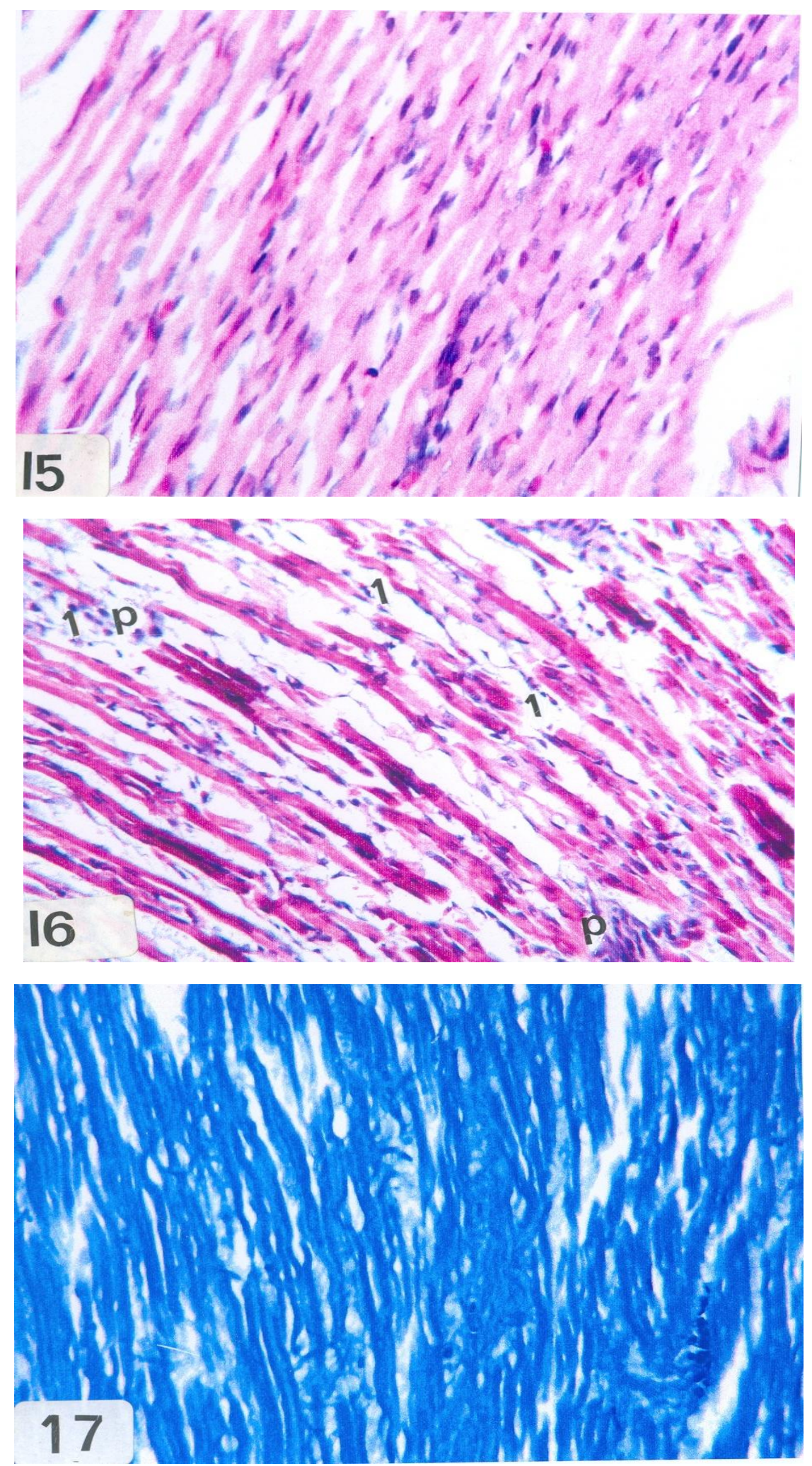

Fig.(15):Showing well developed skeletal muscle fibers of a control fetus. (H\&EX200)

Fig.(16):Photpgraph of a section in skeletal muscle fibers of a fetus taken from exposed rat.Notice:discontinous, disturbed and irregular muscle fibers, some nuclei are not surrounded by cytoplasm(1), others accepted dark coloration (pyknosis,p).

(H\&EX200)

Fig.(17):Showing normal proteinic content in the skeletal muscle fibers of a control fetus .

(Mercuric bromophenol blueX200) 


\section{Nora Ahmed Obaid Alkaabi}
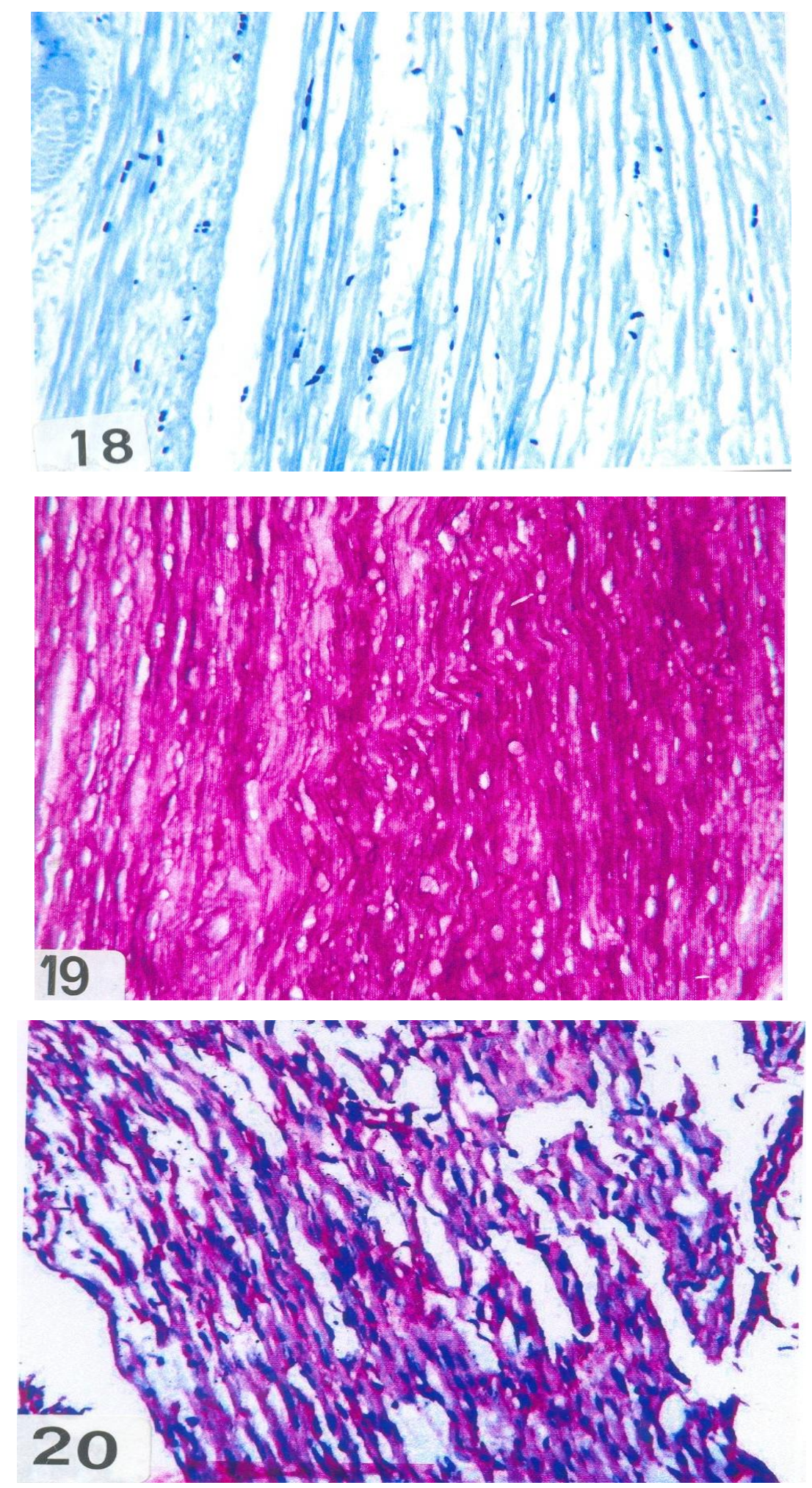

Fig.(18):Showing hypostainability for protein in the muscle fibers and nuclei of their cells, but some nuclei showed hyperstainability.

( Mercuric bromophenol bluex200)

Fig.(19): Showing dense stain ability for polysaccharides in the skeletal muscles of control fetus.

(PASX200)

Fig.(20): Showing highly decreased stain ability for polysaccharides in the skeletal muscles of a fetus taken from an exposed rat

(PASX200) 

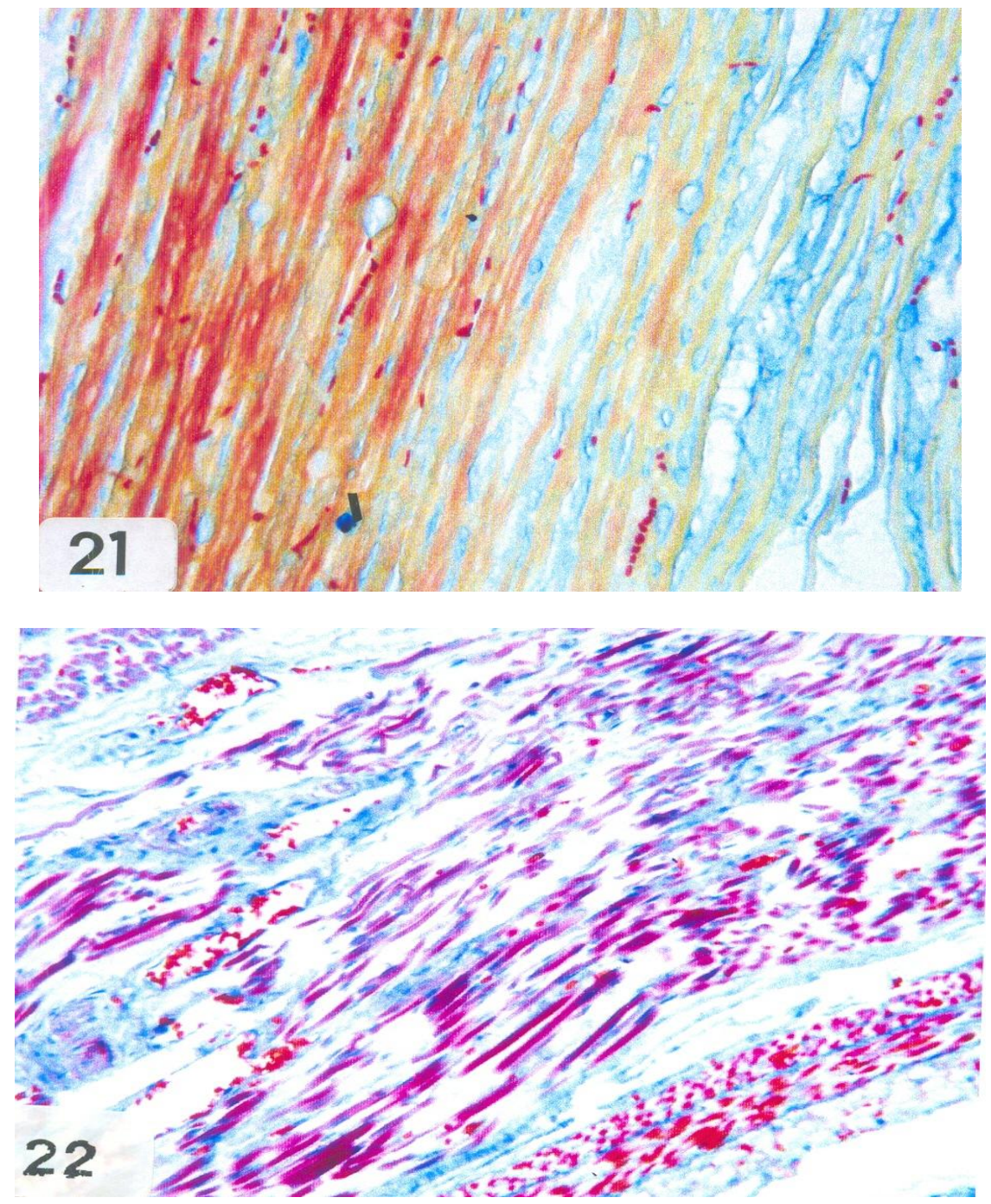

Fig.(21):Showing thin collagenous bundles in between fetal skeletal muscle fibers of the control group (Mallory trichrome stainX200)

Fig.(22):Showing increased collagenous bundles in between the disturbed muscle

\section{Discussion}

(Mallory trichrome stainX200)

There has been increasing interest in the biological effects and possible health outcomes of EMFs. Altered fetal development as a response to EMFs was studied by many authors (Kheifets et al., 2005 \& 2006 and Eid \&Al- Dossary, 2007). Kheifets et al.(2005) reported that the potential vulnerability of children to radiofrequency fields have been raised because of the potentially greater susceptibility to their developing nervous system and their brain tissue which is more conductive .Possible EMF effect on skin was studied by many authors (Imaida et al.,2001; Rajkovic et al., 2005 and
Salem,2008)In the present study many dystrophic changes were observed in the skin and skeletal muscle fibers of fetuses taken from rats exposed to EMF $(50 \mathrm{~Hz} \&$ $2 \mathrm{mT}$ ).Highly thickened epidermal layer (hyperplasia) was observed with many vacuolated cells, some of them contained highly atrophied and deeply stained nuclei Thickened keratin layer was noticed with congested dermal blood vessels and highly reduced hair follicles .Many hemorrhagic areas were detected in the dermal layer.Imaida et al.(2001) detected mouse skin carcinoma post-exposure to $1.5 \mathrm{GHz}$ near fields, while Salem(2008)reported that 


\section{Nora Ahmed Obaid Alkaabi}

exposure of adult male albino rats to EMF from colored TV set ,6hours daily for 2 months caused thinning of all layers of the skin ,hairs were short, fine and easily detached and accepted a yellow color . She also added that apoptosis was observed in the nuclei of the keratinocytes more than that of control group .Hair falling and destruction of skin appendages noticed by Salm(2008)may be due to atrophied and highly reduced hair follicles observed in the present study .Highly dilated dermal blood vessels and presence of many hemorrhagic areas in the dermal layer may be due to the thermal effect of the EMF.In this respect Gorczynska and Wegrzynowicz (1991) stated that the internal bleeding may be due to irregular prothrombin activity postexposure to EMFs.In an attempt to understand the mechanism of action of EMFs on cells ,Dimitris et al.(2002) stated that the basic mechanism is the forced vibration of all the free ions on the surface of plasma membrane of cells ,caused by an external oscillating field .The increased proliferation in the epidermal layer which was observed in the present study was also noted by Detlavs et al .(1996), who observed increased reparative proliferative processes of animals in soft tissues postexposure to EMF.Decreased stain ability for total proteins was observed in the keratin ,epidermal ,dermal and hypodermal layers.Vacuolation observed in many epidermal cells and reduced hair follicles may be responsible for decreased protein content while increased RBCs in many hemorrhagic areas showed intense reaction for total proteins. Highly affected RER,Golgi apparatus and mitochondria in hepatocytes post-exposure to EMF may be responsible for decreased protein content (Eid \& Al-Dossary, 2007) .Highly thickened epidermal layer in the skin of the exposed group showed densely aggregated polysaccharides, while some epidermal areas were thin and poorly stained and so the keratin layer. Decreased polysaccharides in mice skin was noticed by El- Bialy et al.(1995) post- exposure to10 and 14GY gamma rays, and in rat liver post-exposure to EMF $(50 \mathrm{~Hz} \& 2 \mathrm{mT})$ (Eid\&Al-Dossary,2007).They reported that decreased glycogen in hepatocytes postexposure to EMF may be due to failure of hepatocytes to synthesize or store glycogen .Increased polysaccharides in some thickened epidermal areas is in accordance with the results of Gorczynska and Wegrzynowicz (1991).They observed increased glycogen content in hepatocytes post-exposure to EMF, they stated that this increase may be due to increased cortisol which usually leads to an accumulation of glycogen in hepatocytes. Highly thickened keratin, epidermal,dermal and hypodermal layers in skin of the exposed group showed increased collagen fibers and also around the walls of the blood vessels. Thickened collagen fibers post-exposure to EMF from colored TV set was observed by Salem (2008), also ,Fisher et al.(1977) noticed increased collagenase in UV irradiated skin .Increased collagen fibers was detected in liver of pregnant rats and their fetuses exposed to EMF(Eid\&Al-Dossary,2007).

Many histological and histochemical changes were observed in the fetal skeletal muscle fibers taken from mothers exposed to EMF. Discontinuous skeletal muscle fibers could be detected with many pyknotic nuclei and vacuolated or completely degenerated cytoplasm. This degeneration was accompanied by decreased protein content .This hypostainability may be due to damaged DNA (Cheng \& Zou, 2006), increased action of lytic enzymes (El-Banhawy et al.,1986) or ruptured cytoplasmic organoids such as mitochondria , ribosomes,RER and cellular membranes (Gorczynska \& Wegrzynowicz, 1991and Eid\&Al-Dossary,2007).According to $\mathrm{Xu}$ et al.(2001) field intensity of $1 \mathrm{mT}$ might be considered as a threshold level for enhancing muscle microcirculation .Highly decreased polysaccharides was observed in fetal skeletal muscle fibers of the exposed group .The decreased polysaccharides observed in the present study may be due to the increased vacuolation and degeneration observed in the skeletal muscle fibers. Increased collagen fibers could be observed in between and around the disturbed skeletal muscle fibers and in and around the walls of the dilated blood vessels. Pronounced accumulation of collagenous proteins was noticed in muscle fibers of rats exposed to EMF with decreased amounts of glycoproteins macromolecules (Detlvas et al.,1996) .In this respect Karinen et 
al.(2008) suggested that protein expression in human skin might be affected by the exposure to EMF and they added that molecular level changes might take place in human volunteers in response to EMF.The highly dilated dermal blood vessels and sometimes ruptured ones may lead to presence of many hemorrhagic areas .In this respect ,The proteomics studies of Karinen et al.(2008) showed that changes in protein expression and activity (phosphorylation )were induced in human endothelial cell line post-exposure to EMF.

\section{Conclusion}

Results of the present study showed that skin and skeletal muscle fibers of fetuses taken from rats exposed to EMF $(50 \mathrm{~Hz} \& 2 \mathrm{mT})$ are highly sensitive to EMF exposure .Many histological and histochemical changes were detected in skin and skeletal muscle fibers of fetuses taken from exposed rats.

\section{References}

1. Bancroft J D and Gamble M (2002): Theory and Practice of Histological Techniques. $5^{\text {th }}$ ed. Churchill livingstone. London.

2. Cheng $K$ and Zou $C$ (2006) : Electromagnetic field effect on separation of nucleotide sequences and unwinding of a double helix during DNA replication. Med. Hypotheses, 66 (1):148-153.

3. Detlavs I,Dombrovska L,Turauska A, Shkirmante B andSlutskii L(1996): Experimental study of the effects of radiofrequency electromagnetic fields on animals with soft tissue wounds .Sci. Total Environ., 180(1):35-42.

4. Dimitris $\mathbf{J}$, Andreas $\mathbf{K}$ and Lukas $\mathbf{H}$ (2002): Mechanism for action of electromagnetic fields on cellsBiochem. Biophy. Res.Com.,298:95-102.

5. Eid F and Al-Dossary A A (2007): Ultrastructural, histological and histochemical study on the effect of electromagnetic field on the liver of pregnant rats and their fetuses. The Egyptian Journal of Hospital Medicine , 28:273-294.

6. El-Banhawy $M$ A,Alahaby $\mathbf{E}$ and Shalaby A A(1986): Effect of cyolane intoxification of the protein contents in epithelial cells of Clarias lazera Egypt.J . Histol.,9(1):69-76.

7. El-Bialy M, El-Salkh B,El-Asser,A and Hafez M(1995):Chemical protection of
Albino mice against Histochemical changes in certain skin carbohydrates affected by shot and intermittent whole body Gamma irradiation .J.Egypt.Ger.Soc.Zool. ,18:125152.

8. Fisher G, Wang Z,Datta $\mathbf{S}$ and Varani J (1977): pathophysiology of premature skin aging induced by ultraviolet light .N.Engl. J.Med. ,337:1419-1426.

9. Gorczynska, E and Wegrzynowicz, R. (1991): Structural and functional changes in organelles of liver cells on rat exposed to magnetic fields.Environ. Res.,55:188 - 189.

10. Hassan $M$ and Sorour $\mathbf{H}$ (2006): Human Histology .Commercial Press Kalyou. Cairo.

11. Hotchkiss R D (1948): A microchemical reaction resulting in the staining of polysaccharide structures in fixed tissue preparations. Arch. Biochem.., 16: 131-132.

12. Imaida K, Kuzutani K,Wang J,Fujiwara O,Ogiso T, Kato $K$ and Shirai $T$ (2001) :Lack of promotion of 7,12 -dimethylbenz(a) anthracene-initiated mouse skin carcinogenesis by $1.5 \mathrm{GHz}$ electromagnetic near fields.Carcinogenesis,22(11):1837-1841.

13. Karinen A,Heinavaara $S$, Nylund $R$ and Leszzynski D (2008): Mobile phone radiation might alter protein expression in human skin .B .M .C . Genomics, 11:9-77.

14. Kheifet L, Repacholi M , Saunders R and van Deventer $E$ (2005): The sensitivity of children to electromagnetic fields. Pediatrics 116(2): 303-313.

15. Mazia D, Brewer PA and Alfert $M$ (1953): The cytochemical staining and measurement of protein with mercuric bromophenol blue. Biol. Bull., 104:57-67.

16. Pearse, A.G. (1977): Histochemistry , Theoretical, and Applied. $3^{\text {ed }}$, Vol. I. Churchill Livingstone, London.

17. Rajkovic V,Matavulj $M$ and Johnsson $\mathbf{O}$ (2005) :The effect of extremely lowfrequency electromagnetic fields on skin and thyroid amine - and peptide-containing cells in rats : an immunohistochemical and morphometrical syudy. Environ. Res., 99 (3): 369-377.

18. Salem M (2008) : Protective effect of Vitamin A against skin injury caused by exposure to electromagnetic field .Histological and Histochemical studies. The Egyptian Journal of Hospital Medicine,

19. Xu S, Okano H and Ohkuvo C (2001): Acute effects of whole -body exposure to static magnetic fields and $50-\mathrm{Hz}$ electromagnetic fields on muscle microcirculation in anesthetized mice.Bioelectrochemist. 53:127-135. 
تأثير المجال الكهرومغناطيسى على العضلات الهيكلية وجلد أجنة الجرذان البيضاء البفلات

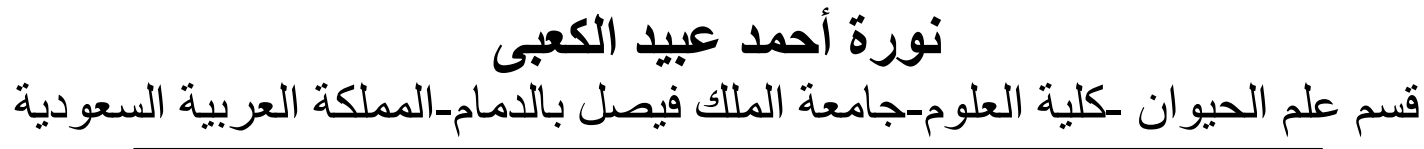

تضمنت هذه الدر اسة تأثير التعرض لمجال مغناطيسى ذو نردد50 هرتزو كثنافية

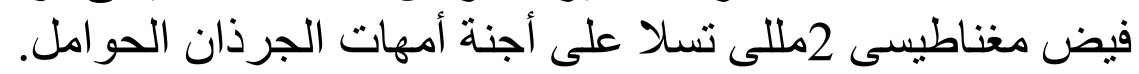

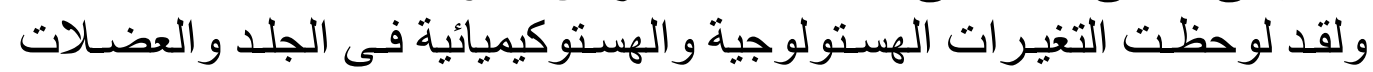

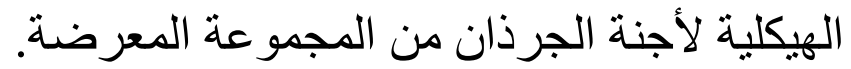

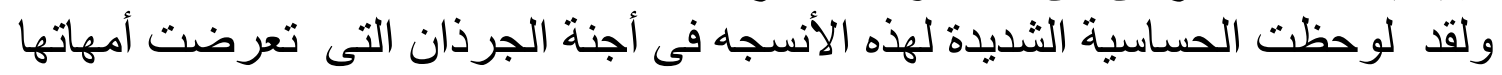

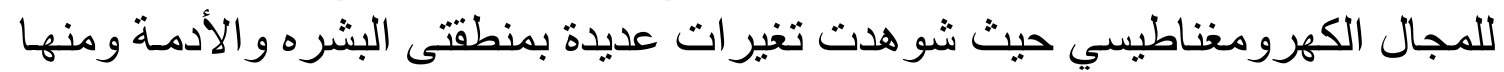

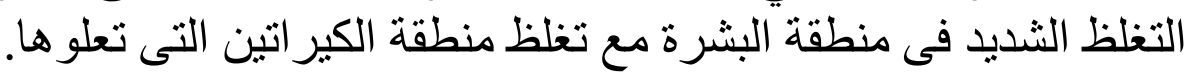

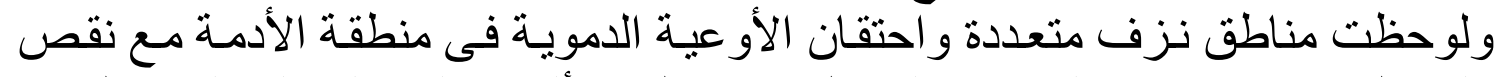

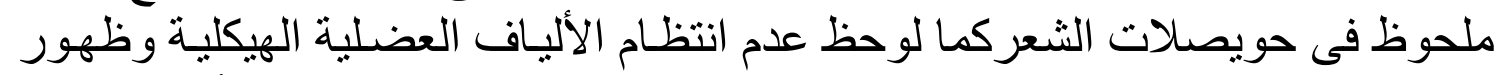

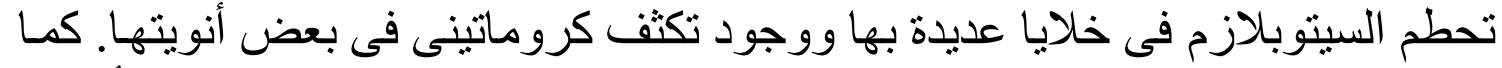

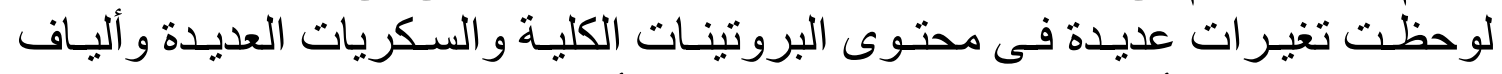
الكو لاجين فى جلد أجنة الجرذان المعرضة وكذللك ألياف العضلات الهيكلية لها. 